\title{
Modelado neuronal de un proceso de digestión aeróbica de aguas residuales
}

\author{
Neuronal modeling of a process of aerobic digestion of wastewater
}

\section{Roxana B. Recio Colmenares.; Kelly J. Gurubel Tún}

Departamento de Estudios del Agua y la Energía, CUTONALA, Universidad de Guadalajara,Avenida Nuevo Periférico No. 555, Ejido San José Tatepozco, Tonalá, Jalisco, 45425, México.joel.gurubel@cutonala.udg.mx, recio.roxana@gmail.com

\section{Abstract}

Los procesos biológicos para el tratamiento de agua residual son sistemas altamente no lineales que están sometidos a incertidumbres y perturbaciones externas, se ha creído ampliamente que los modelos que describen las reacciones bioquímicas y procesos de sedimentación son muy complejos y difíciles de manejar exactamente con fines de control. Por tal motivo se requieren estrategias avanzadas para la identificación, estimación de parámetros no medibles del proceso y control.En este trabajo se propone una red neuronal no lineal en tiempo discreto en presencia de perturbaciones externas $y$ estimación de los estados dinámicos difíciles de medir en un proceso de digestión aerobia para tratamiento de agua residual. Se presentan resultados de estimación vía simulación, donde se demuestra que el modelo neuronal propuesto es eficiente para la estimación de las dinámicas de los estados en presencia de perturbaciones. Con este modelo neuronal será posible diseñar una estrategia de control para optimizar el rendimiento de la degradación de materia orgánica en un proceso de digestión aerobia usando técnicas de control óptimo y estimación neuronal.

Keywords- Modelo ASM1, Red neuronal, dinámicas no lineales, agua residual.

\section{INTRODUCTION}

Los modelos de lodos activados (ASM), son metodologías avanzadas que representan el "estado de arte" aplicadas en procesos de lodos activados. Los modelos ASM1 y ASM3 son capaces de simular la remoción de materia orgánica, nitrificación y desnitrificación [1].

Este modelo es una norma internacionalmente aceptada para el modelado de lodos activados y es utilizado ampliamente en las plantas de tratamiento de agua residual para reducir los niveles de contaminación de materia orgánica procedente de los sectores municipal e industrial, basada en el consumo de la demanda biológica de oxigeno (DBO) [2].

Se ha creído ampliamente que los modelos complejos que describen reacciones bioquímicas y procesos de sedimentación son muy difíciles de manejar exactamente con fines de control. El modelado matemático ha demostrado ser una herramienta poderosa 
164

para ayudar a comprender el impacto de estas condiciones dinámicas de influente en el rendimiento general del proceso de la planta.

De igual forma, se han mejorado aspectos en cuanto a avances de investigación en las interacciones entre el sistema de alcantarillado y la planta de tratamiento de aguas residuales y receptores de aguas, avances de investigación en la relación entre el estado ecológico y el estado físico - químico de las aguas receptoras; y la disponibilidad de software que permite el uso de modelos integrados [4].

\section{MODELO MATEMATICO}

Este modelo cuenta con 13 componentes (7 con materia orgánica soluble " $S$ " y 6 con particulada " $X$ "). De los 13 componentes, 7 son compuestos carbonosos : 2 solubles (SI y SS) y 5 particula-dos (XI, XS, XBH, XBA y XP), otros 4 son compuestos nitrogenados: 3 solubles (SNO, SNH y SND) y 1 particulado (XND). Solo las fracciones biodegradables soluble SND y particulada XND se consideran explícitamente en el modelo [1].

El modelo ASM1 que describe la dinámica del reaccionante en un estudio determinado, consta de ecuaciones diferenciales ordinarias (ODEs) no lineales que representan los balances de materia de los componentes. Dado que se asume sistema isotérmico se prescinde del balance de energía (suposición generalmente aceptada en el modelado de sistemas de lodos activados). De esta manera, la complejidad depende hasta cierta medida de la cantidad de ODEs necesarias para describir el comportamiento del sistema [5].
El modelo reducido consta de 9 ecuaciones diferenciales ordinarias que describen el proceso de transformación bilógico. Las variables de estado que describen las concentraciones se describen en la tabla 1.

$$
\begin{aligned}
& \frac{d S s}{d t}=\frac{q}{V}=\left(S s,{ }_{\text {in }}-S s\right)-\frac{1}{Y H} \cdot \mu_{\max , H} \cdot M_{2} \cdot\left(M_{8 h}+I_{8} \cdot M_{9} \cdot n_{g}\right) . \\
& X_{B H}+k_{h} \cdot k_{s a t}\left(M_{8 h}+n_{h} \cdot I_{8} \cdot M_{9}\right) X_{B H} \cdot \\
& \frac{d X s}{d t}=\frac{q}{V}\left(X s,_{\text {in }}-X s\right)+\frac{q r}{V}(b-1) X s+(1-f p) \\
& \left(b_{H} X_{B, H}+b_{A} X_{B A}\right)-k_{h} \cdot k_{s a t}\left(\mathrm{M}_{8 H}+n_{h} \cdot I_{8} \cdot M_{9}\right) X_{B ; H} \cdot \\
& \frac{d X_{B, H}}{d t}=\frac{q}{V}\left(X_{B, H, \text { in }}-X_{B, H}\right)+\frac{q r}{V}(b-1) X_{B, H}+\mu_{\max , H} \cdot \\
& M_{2} \cdot M_{8 h} \cdot X_{B, H}+\mu_{\max , H} \cdot M_{2} \cdot I_{8} \cdot M_{9} \cdot \mathrm{ng} \cdot X_{B, H}-b_{H} \cdot X_{B, H} \cdot
\end{aligned}
$$

$\frac{d X_{B ; A}}{d t}=\frac{q}{V}\left(X_{B, A, i n}-X_{B ; A}\right)+\frac{q r}{V}(b-1) X_{B, A}+\mu_{M A X, A} M_{10} M_{8 a} \cdot X_{B A}-b_{A} \cdot X_{B, A}$.

$$
\begin{aligned}
& \frac{d S o}{d t}=\frac{q}{V}\left(S o,_{\text {in }}-S o\right)+K_{L, A}\left(S o,,_{\max }-S o\right)-\frac{\left(1-Y_{H}\right)}{Y_{H}} \\
& \mu_{\max \cdot H} \cdot \mathrm{M}_{2} \cdot M_{8 H} \cdot X_{B, H}-\frac{\left(4.57-Y_{A}\right)}{Y_{A}} \cdot \mu_{\max , \mathrm{A}} \cdot M_{10} \cdot M_{8 a} \cdot \mathrm{X}_{B, H} \cdot
\end{aligned}
$$

$$
\begin{aligned}
& \frac{d S_{N O}}{d t}=\frac{q}{V}\left(S_{N O, \text { in }}-S_{N O}\right)-\frac{\left(1-Y_{H}\right)}{2 \cdot 86 Y_{H}} \cdot \mu_{\operatorname{maxH}} \cdot M_{2} \cdot I_{8} \cdot M_{9} . \\
& n_{g} \cdot X_{B, H}+\frac{1}{Y_{A}} \cdot \mu_{M A X, A} \cdot M_{10} \cdot M_{8 a} \cdot X_{B, \mathrm{~A} .}
\end{aligned}
$$

$$
\begin{aligned}
& \frac{d S_{N H}}{d t}=\frac{q}{V}\left(S_{N H, i n}-S_{N H}\right)-i_{X B} \mu_{\max , H} \cdot M_{2}\left(M_{8 h}+I_{8} \cdot \mathrm{M}_{9} \cdot n g\right) X_{B, H} \\
& -\left(i_{X B}+\frac{1}{Y_{A}}\right) \mu_{\max , A} \cdot M_{10} \cdot M_{8 a} \cdot X_{B, A}+k_{A} \cdot S_{N D} \cdot X_{B, H} .
\end{aligned}
$$


$\frac{d S_{N D}}{d t}=\frac{q}{V}\left(S_{N D, \text { in }}-S_{N D}\right)-k_{A} . S_{N D} \cdot X_{B, H}+k_{h}$

$k_{\text {sat }}\left(M_{8 h}+n_{h} I_{8} M_{9}\right) X_{B, H} \frac{X_{N D}}{X_{S}}$.

$\frac{d X_{N D}}{d t}=\frac{q}{V}\left(X_{N D, \text { in }}-X_{N D}\right)+\frac{q_{r}}{V}(b-1) X_{N D}+\left(i_{X B}-f_{p} \cdot i_{X P}\right)\left(b_{H} \cdot X_{B, H}+b_{A} \cdot X_{B, A}\right)$

$-k_{h} \cdot k_{s a t} \cdot\left(M_{8 h}+n_{h} \cdot I_{8} \cdot M_{9}\right) X_{B, H} \cdot \frac{X_{N D}}{X_{S}}$

Las velocidades de reacción correspondientes son:

$M_{2}=\frac{S_{S}}{K_{S}+S_{S}}$

$M_{8 a}=\frac{S_{O}}{K_{O, A}+S_{O}}$

$M_{8 h}=\frac{S_{O}}{K_{O, H}+S_{O}}$

$M_{9}=\frac{S_{N O}}{K_{N O}+S_{N O}}$

$M_{10}=\frac{S_{N H}}{K_{N H}+S_{N H}}$,

$I g=\frac{K_{O, H}}{K_{O, H}+S_{O}}$

$k_{s a t}=\frac{X_{S}}{K_{X} X_{B, H}+X_{S}}$,

\begin{tabular}{|c|c|}
\hline Componente & Definición \\
\hline 1. $\mathrm{S}_{\mathrm{I}} \mathrm{M}(\mathrm{COD}) \mathrm{L}^{-3}$ & $\begin{array}{l}\text { Materia orgánica inerte } \\
\text { soluble }\end{array}$ \\
\hline 2. $\mathrm{Ss} \mathrm{M}(\mathrm{COD}) \mathrm{L}^{-3}$ & $\begin{array}{l}\text { Sustrato rápidamente } \\
\text { biodegradable }\end{array}$ \\
\hline 3. $\mathrm{X}_{\mathrm{I}} \mathrm{M}(\mathrm{COD}) \mathrm{L}^{-3}$ & $\begin{array}{l}\text { Materia orgánica en } \\
\text { partículas inerte }\end{array}$ \\
\hline 4. Xs M(COD) $\mathrm{L}^{-3}$ & $\begin{array}{l}\text { Sustrato lentamente } \\
\text { biodegradable en partícula }\end{array}$ \\
\hline 5. $\mathrm{X}_{\mathrm{B}, \mathrm{H}} \mathrm{M}(\mathrm{COD}) \mathrm{L}^{-3}$ & $\begin{array}{l}\text { Biomasa heterotrófica } \\
\text { activa }\end{array}$ \\
\hline 6. $\quad \mathrm{X}_{\mathrm{B}, \mathrm{A}} \mathrm{M}(\mathrm{COD}) \mathrm{L}^{-3}$ & autotrófica \\
\hline 7. $\mathrm{Xp} \mathrm{M(COD)} \mathrm{L}^{-3}$ & $\begin{array}{l}\text { Productos provenientes } \\
\text { del decaimiento de la } \\
\text { biomasa }\end{array}$ \\
\hline 8. $\mathrm{S}_{\mathrm{O}} \mathrm{M}(-\mathrm{COD}) \mathrm{L}^{-3}$ & Oxígeno disuelto \\
\hline 9. $\mathrm{S}_{\mathrm{ON}} \mathrm{M}(\mathrm{N}) \mathrm{L}^{-3}$ & $\begin{array}{lll}\text { Nitratos } & \text { y } & \text { Nitritos } \\
\text { solubles } & & \\
\end{array}$ \\
\hline 10. $\mathrm{S}_{\mathrm{NH}} \mathrm{M}(\mathrm{N}) \mathrm{L}^{-3}$ & Amoniaco soluble \\
\hline 11. $\mathrm{S}_{\mathrm{ND}} \mathrm{M}(\mathrm{N}) \mathrm{L}^{-3}$ & $\begin{array}{l}\text { Nitrógeno orgánico } \\
\text { soluble rápidamente } \\
\text { biodegradable }\end{array}$ \\
\hline 12. $\mathrm{X}_{\mathrm{ND}} \mathrm{M}(\mathrm{N}) \mathrm{L}^{-3}$ & $\begin{array}{l}\text { Nitrógeno orgánico en } \\
\text { partícula biodegradable }\end{array}$ \\
\hline $\begin{array}{l}\text { 13. } \mathrm{S}_{\mathrm{ALK}} \text { (unidades } \\
\text { molares) }\end{array}$ & Alcalinidad \\
\hline
\end{tabular}


166

Las redes neuronales artificiales se han utilizado con éxito en procesos biológicos para el modelado, la predicción y el control de dinámicas no lineales [6]. Las Redes Neuronales Recurrentes (RHONN) han demostrado ser factibles en aplicaciones de identificación y control debido a su arquitectura flexible y robustez [7].

El observador de intervalo [8], [9] es una buena alternativa para resistir los efectos de las incertidumbres del sistema; sin embargo, la razón de la convergencia de la estimación no puede estar en sintonía. Además, se puede inducir un efecto de sobreestimación en los intervalos considerados. Por otro lado, el observador propuesto en [10] está dedicado a la estimación de las entradas no medidas además de las variables de estado desconocido; se basa en un diseño de linealización tangencial, que puede limitar el rango de operación del proceso. Finalmente, observadores neuronales basados en Recurrent High Order Neuronal Network (RHONN) para el proceso anaeróbico de tratamiento de agua se proponen en [11], [12].

Este observador se basa en una Recurrent High Order Neuronal Network (RHONN) en tiempo discreto las cuales incluyen no sólo las combinaciones lineales de sus componentes, sino que además consideran sus productos, lo cual permite obtener interacciones de alto orden.

Esta red es entrenada con un algoritmo basado en un Extendend Kalman Filter (EKF). [13-16], el objetivo filtro de Kalman es diseñar un observador óptimo que estime los estados de un sistema con ruido blanco en la salida y en los estados.

Considerando el sistema discreto siguiente:

$$
\begin{aligned}
& y(k)=C x(k)+v_{1}(k) \\
& x(k+1)=A x(k)+B u(k)+u_{2}(k)
\end{aligned}
$$

Donde $v_{1}(k)$ y $v_{2}(\mathrm{k})$ es ruido blanco, es decir, la medida del ruido es 0 , esto quiere decir que la esperanza es $0 \quad\left(E\left\{v_{1}(k)=0\right\}\right)$.

La estructura del sistema es esencialmente una copia del sistema, entonces se supone la estructura del observador.

Las redes neuronales recurrentes han demostrado ser factibles en aplicaciones de identificación y control debido a su arquitectura flexible y robustez [10]. Las redes neuronales recurrentes de alto orden discretas tienen las mismas características que las continuas. A partir de la discretización se obtiene el modelo discreto de una RHONN.

$x(k+1)=w_{i}^{T} z(x(k), u(k)), i=1, \ldots, \mathrm{n}$

Donde $x_{i}(k)$ es el estado de la i-ésima neurona, en la iteración $k, w_{i}$ es el vector de pesos sinápticos de la red neuronal, estos son adaptados en línea; $n$ representa la dimancion del estado; $z(x(k), u(k))$, es el vector de entrada a cada neurona, y finalmente $u$ que representa el vector de entradas a la red neuronal y $S($.) una función sigmoidal que se define acontinuación.

$$
S(x)=\frac{\mu}{1+e^{-\beta x}}+\varepsilon
$$

Dónde: 
167

$\mu$ Es la cota de la sigmoidal

$\beta$ Es la pendiente de la curva sigmoidal

$\varepsilon$ Es el número real positivo que desplaza la sigmoidal.

Se han propuesto algunos observadores que estimen las variables difíciles de medir, aplicados en general a procesos biotecnológicos, todos estos observadores tienen buenos resultados, sin embargo, algunos de ellos, presentan dificultades en el diseño e implementación, errores de estimación dadas las incertidumbres del modelo, etc., y además requieren contar con el modelo dinámico del proceso, lo cual no es siempre posible restringiéndose de esta forma el diseño.

Dado lo anterior, en este trabajo se propone el uso de un observador neuronal discreto, el cual no requiere del conocimiento del modelo del proceso. Este nuevo observador se basa en la aproximación a partir de la medición de las salidas. Para el sistema se propone un observador neuronal tipo Luenberger con la siguiente estructura:

$$
\begin{aligned}
& \hat{x}_{i}(k+1)=w_{i}^{T} z_{i}(\hat{x}(k), u(k))+L_{i} e(k), i=1, \ldots, n \\
& \hat{Y}(k)=h(\hat{x}(k))
\end{aligned}
$$

Donde $z_{i}(\hat{x}(k), u(k))$ es el vector de entrada a cada neurona, $w_{i}$ es el vector de pesos sinápticos de la red neuronal y $L_{i} \in \square^{1 x p}$.

El sistema no lineal discreto general puede ser aproximado como una RHONN discreta con representación en paralelo, la cual se supone observable y está dado como sigue:

$x_{i}(k+1)=w_{i}^{* T} Z_{i}(x(k), u(k))+\epsilon_{z i}, i=1, \ldots, n$

Donde $x_{i}$ es el i-ésimo estado de la planta, $\epsilon_{z i}$, es el error de aproximación, este error puede ser reducido mediante el incremento de numero de pesos. Se supone que existe un vector ideal de pesos $w_{i}^{*}$ tal que $\left\|\in_{z i}\right\|$ pueda ser minimizada en un conjunto compacto $\Omega_{z i} \subset \square^{L i}$ (2) en general se supone que este vector existe, es constante pero desconocido si se define $w_{i}$ como la estimación de $w_{i}^{*}$, entonces el vector de estimación de pesos está dado por:

$\tilde{w}_{i}(k)=w_{i} *-w_{i}(k)$

\section{Estructura del identificador}

Se propone un identificador neuronal de tiempo discreto para cada estado del modelo ASM1; para limitaciones de la longitud, solo los primeros tres modelos de identificador neuronal se incluyen de la siguiente manera:

$$
\begin{aligned}
& \hat{Z}(k+1)=W_{1} S\left(Z_{1}(k)\right)+W_{2} S\left(Z_{2}(k)\right)+W_{3} S\left(Z_{3}(k)\right)+ \\
& W_{5} S\left(Z_{5}(k)\right)+W_{6} S\left(Z_{6}(k)\right) \\
& \hat{Z}_{2}(k+1)=W_{2} S\left(Z_{2}(k)\right)+W_{3} S\left(Z_{3}(k)\right)+W_{4} S\left(Z_{4}(k)\right)+ \\
& W_{5} S\left(Z_{5}(k)\right)+W_{6} S\left(Z_{6}(k)\right) \\
& \hat{Z}_{3}(k+1)=W_{1} S\left(Z_{1}(k)\right)+W_{3} S\left(Z_{3}(k)\right)+W_{5} S\left(Z_{5}(k)\right)+ \\
& W_{6} S\left(Z_{6}(k)\right) \\
& \hat{Z}_{4}(k+1)=W_{4} S\left(Z_{4}(k)\right)+W_{5} S\left(Z_{5}(k)\right)+W_{7} S\left(Z_{7}(k)\right) \\
& \hat{Z}_{5}(k+1)=W_{3} S\left(Z_{3}(k)\right)+W_{4} S\left(Z_{4}(k)\right)+W_{5} S\left(Z_{5}(k)\right)
\end{aligned}
$$


$\hat{Z} 6(k+1)=W_{3} S\left(Z_{3}(k)\right)+W_{4} S\left(Z_{4}(k)\right)+W_{6} S\left(Z_{6}(k)\right)$

$\hat{Z} 7(k+1)=W_{3} S\left(Z_{3}(k)\right)+W_{4} S\left(Z_{4}(k)\right)+W_{7} S\left(Z_{7}(k)\right)+$

$W_{8} S\left(Z_{8}(k)\right)$

$\hat{Z} 8(k+1)=W_{2} S\left(Z_{2}(k)\right)+W_{3} S\left(Z_{3}(k)\right)+W_{8} S\left(Z_{8}(k)\right)$

$\hat{Z} 9(k+1)=W_{2} S\left(Z_{2}(k)\right)+W_{3} S\left(Z_{3}(k)\right)+W_{4} S\left(Z_{4}(k)\right)+$

$W_{9} S\left(Z_{9}(k)\right)$

Para comprobar la robustez del sistema se variaron las entradas considerando una función de activación, la cual se caracteriza por definir las salidas de las neuronas en términos de potencial de activación

La función de activación tipo escalón se muestra en la ecuación 31 donde

$$
\varphi(v)=\left\{\frac{1 \operatorname{siv} \geq 0}{0 \operatorname{siv} \prec 0}\right.
$$

La salida de la neurona $\mathrm{n}$ está definida por la siguiente ecuación

$$
y_{n}=\left\{\begin{array}{l}
1 \text { si } v_{n} \geq 0 \\
0 \text { si } v_{n}<0
\end{array}\right.
$$

La función sigmoidea es la más usada en redes neuronales, la síguete ecuación muestra un ejemplo de una función sigmoidea descrita a continuación:

$$
\varphi(v)=\frac{1}{1+e^{-a v}}
$$

Para la función lineal a tramos se muestra en la siguiente

\section{Ecuación}

$$
\varphi(v)=\left\{\begin{array}{rlrl}
1 & v & \geq+\frac{1}{2} \\
v & +\frac{1}{2}>v & >-\frac{1}{2} \\
0 & v & \geq+\frac{1}{2}
\end{array}\right.
$$

168

\section{RESULTADOS DE SIMULACION}

Se presentan los resultados de la simulación, donde se demuestra que el modelo neuronal es eficiente para calcular la dinámica compleja del proceso en presencia de perturbaciones. Como trabajo futuro, se pueden desarrollar algoritmos de control y optimización basada en el modelo neuronal para optimizar la degradación de la materia orgánica en primera instancia, también se propone la optimización energética considerando el control óptimo de un sistema de aeración.

En el esquema propuesto se plantea la identificación de las dinámicas de los microrganismos y sustratos difíciles de medir en el proceso y que están relacionados directamente con la degradación de la materia orgánica.

Como se puede observar la red neuronal identifica correctamente la dinámica compleja del sistema de digestión aerobia.

La contribución que las ciencias computacionales pueden tener la solución de problemas complejos referidos a la calidad y cantidad del agua, se ha constituido como una alternativa de gran importancia en la toma de decisiones informada y en la producción de conocimiento.

Sobre esta base disciplinar, actualmente, existe la oportunidad de desarrollar sistemas de información computacionales desarrollados bajo el enfoque sistemas dinámicos y de inteligencia artificial, que pueden ser integrados con herramientas WEB, tecnologías 
169

de automatización y control, y tecnologías telemáticas [18].

La identificación de lleva a cabo con las respuestas dinámicas del modelo matemático sometido a perturbaciones externas incrementables considerando como entrada al sistema el KLA en un periodo de prueba de 96 horas.

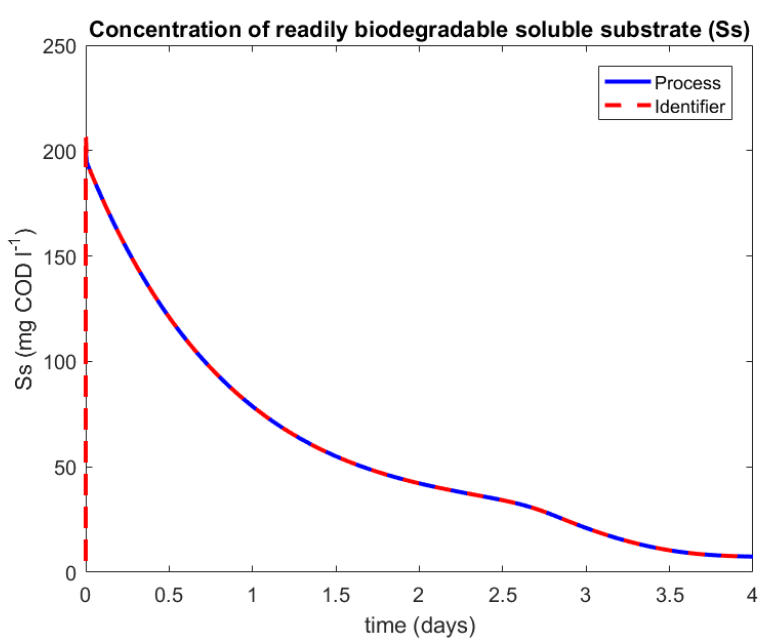

Fig. 1 Concentración de sustrato soluble rápidamente biodegradable.

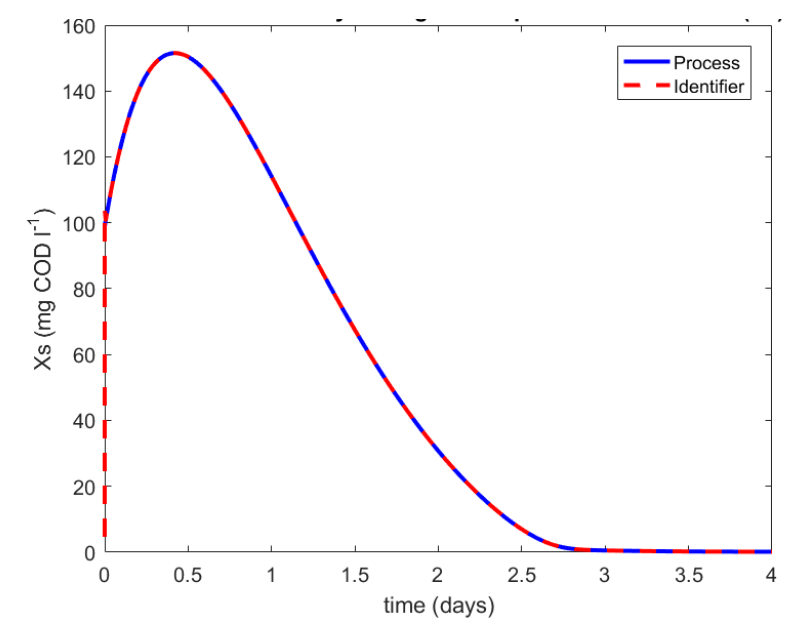

Fig. 2 Concentración de sustrato soluble lentamente biodegradable $(X s)$.

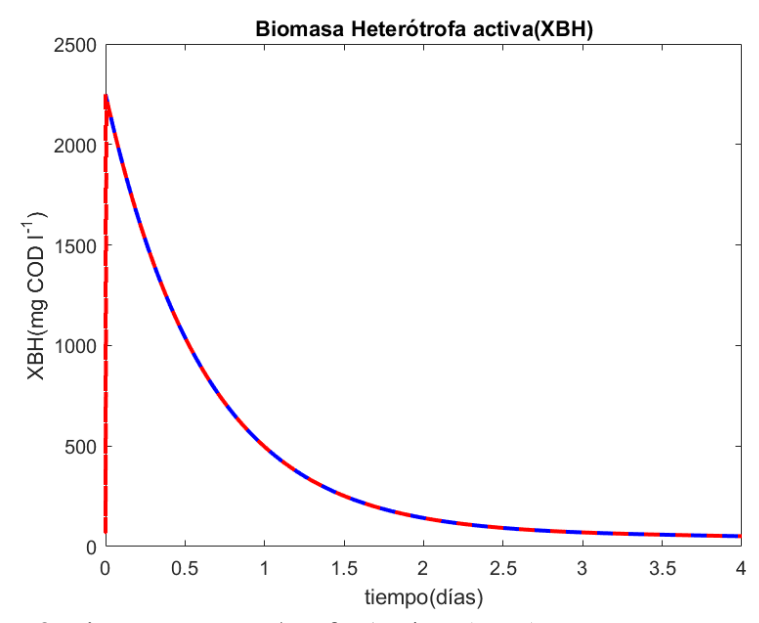

Fig. 3 Biomasa Heterótrofa Activa $\left(\mathrm{X}_{\mathrm{BH}}\right)$.

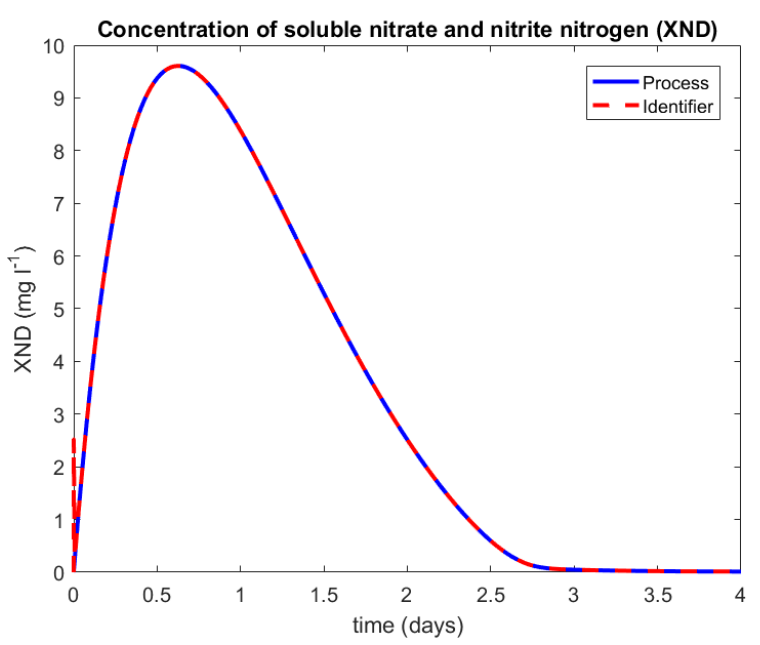

Fig. 3 Concentración de nitrato y nitrito

La red neuronal utilizada en la simulación identifica eficientemente la dinámica del proceso de digestión aerobia para los sustratos y las biomasas.

El error cuadrático medio (MSE) alcanzado en la identificación de esta red neuronal con un algoritmo EKF demuestra que captura eficientemente la complejidad asociada con la dinámica del proceso. 


\begin{tabular}{|l|l|}
\hline & \multicolumn{1}{|c|}{ MSE } \\
\hline$S_{S}\left(m g C O D l^{-1}\right)$ & $1.4746 e-04$ \\
\hline$X_{S}\left(m g C O D l^{-1}\right)$ & $7.9257 e-06$ \\
\hline$X_{B, H}\left(m g C O D l^{-1}\right)$ & $5.4519 e-04$ \\
\hline$X_{B, A}\left(m g C O D l^{-1}\right)$ & $5.3162 e-05$ \\
\hline$S_{O}\left(\mathrm{mgl}^{-1}\right)$ & $1.3839 e-05$ \\
\hline$S_{N O}\left(m g N l^{-1}\right)$ & $2.1160 e-05$ \\
\hline$S_{N H}\left(m g N l^{-1}\right)$ & $5.8408 e-05$ \\
\hline$S_{N D}\left(m g N l^{-1}\right)$ & $2.2117 e-05$ \\
\hline$X_{N D}\left(m g C O D l^{-1}\right)$ & $3.5578 e-06$ \\
\hline
\end{tabular}

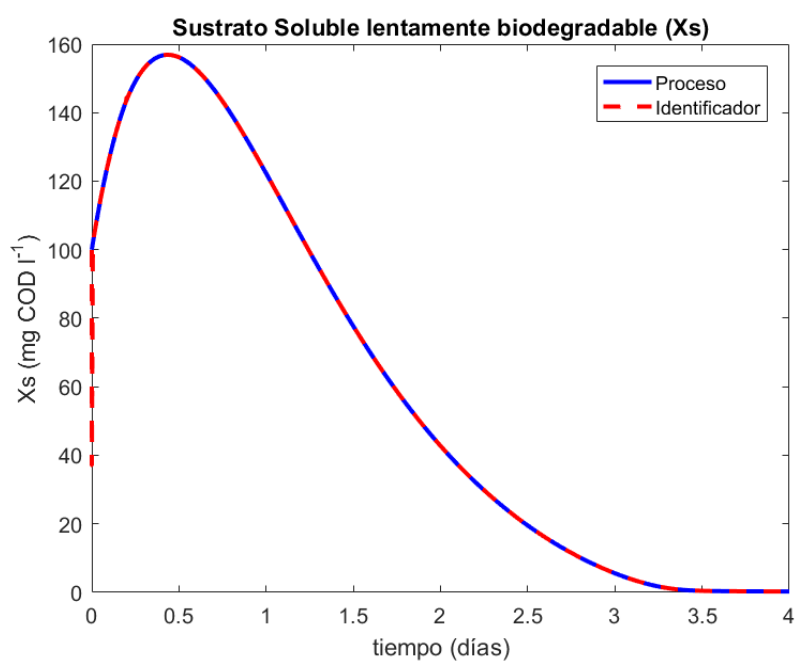

Adicionalmente se simuló el modelo para garantizar la robustez ante los cambios. EL KLA fue variado considerando una entrada escalón para analizar la respuesta ante diferentes entradas.

La siguiente figura muestra el comportamiento del sustratos y biomasas considerando una entrada escalón.

Mostrando resultados satisfactorios con relación a los estados estimados.
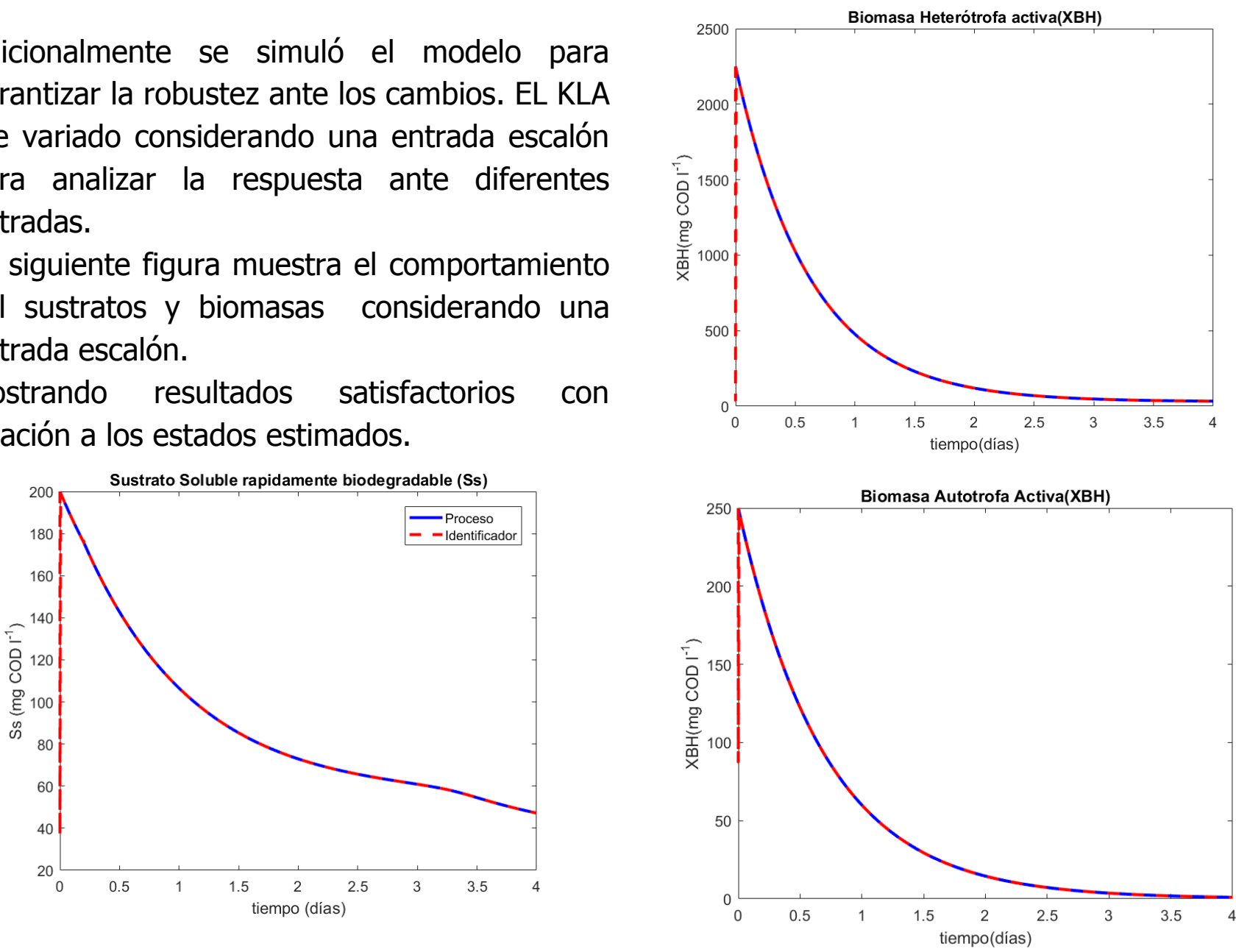

La siguiente tabla muestra el error medio cuadrático considerando una entrada escalón. 


\begin{tabular}{|l|l|}
\hline Proceso & MSE \\
\hline$S_{S}\left(m g C O D l^{-1}\right)$ & 0.0399 \\
\hline$X_{S}\left(m g C O D l^{-1}\right)$ & $2.1753 \mathrm{e}-05$ \\
\hline$X_{B, H}\left(m g C O D l^{-1}\right)$ & 0.0534 \\
\hline$X_{B, A}\left(m g C O D l^{-1}\right)$ & 0.2206 \\
\hline
\end{tabular}

La siguiente simulación muestra la respuesta del sistema y del observador con entrada rampa considerando a KLA como entrada al sistema
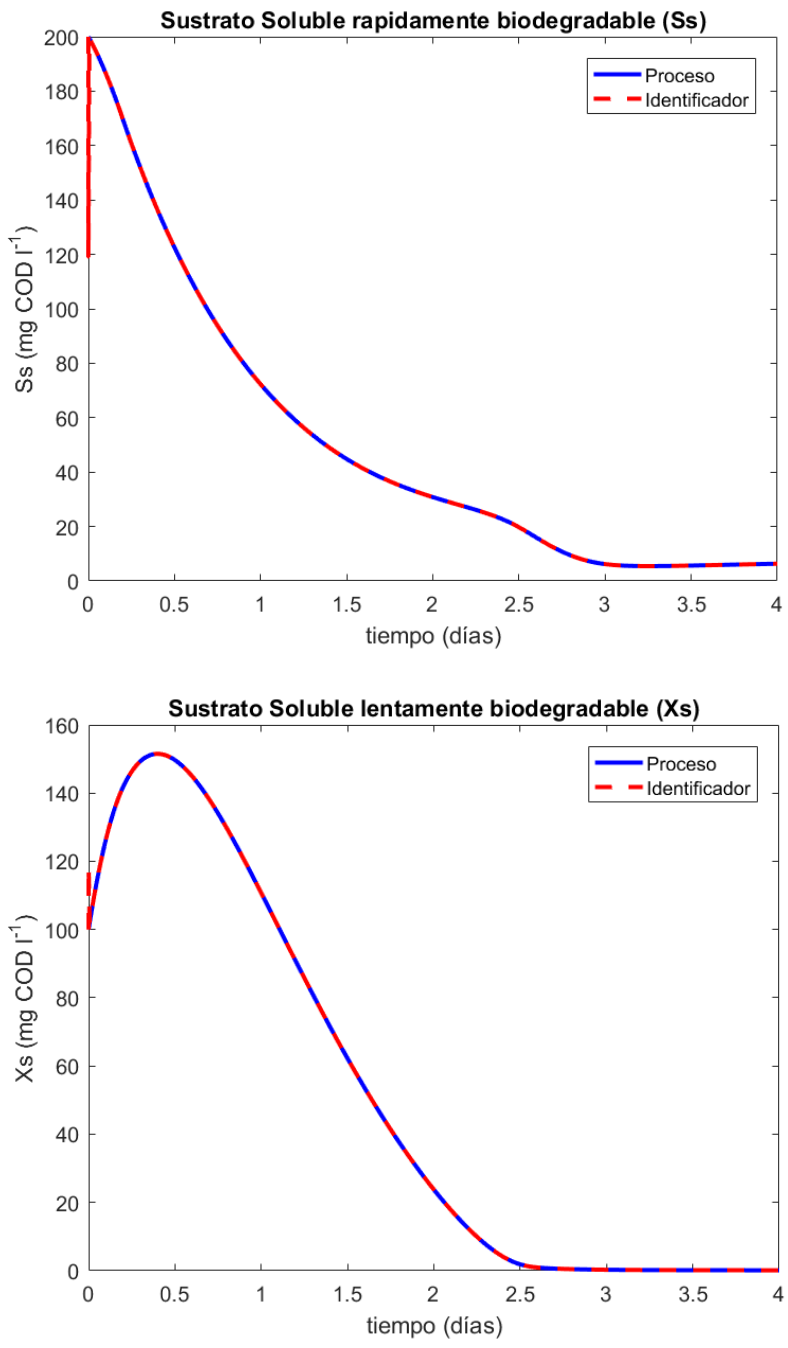

171
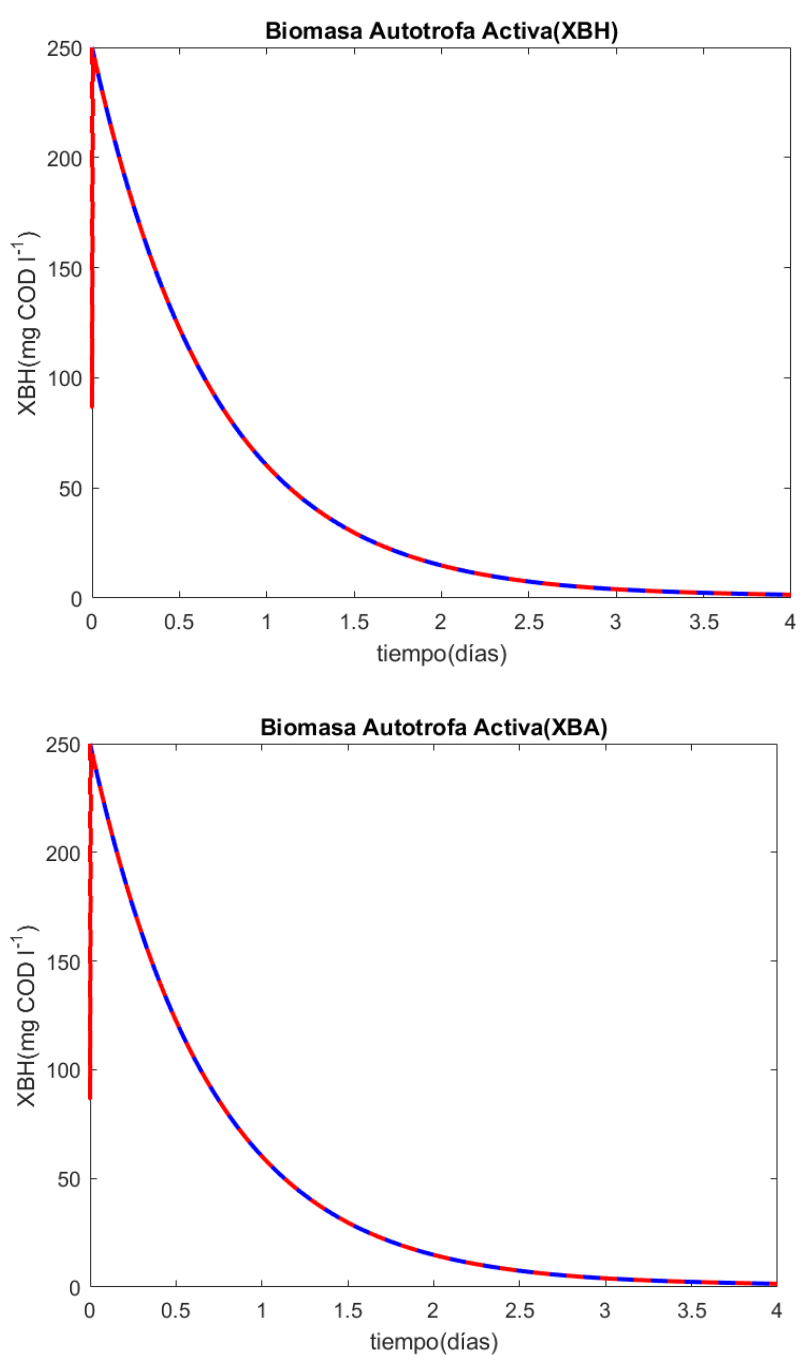

Se puede observar que el observador neuronal actúa correctamente en relación a la dinámica del proceso y su error medio cuadrático.

\section{REFERENCES}

[1] Espinosa-Rodríguez, M. A., \& Fall, C. (2015). Optimization de la producción de lodos en un sistema de lodos activados a través de la 
calibración del modelo ASM1. Ingeniería, investigación y tecnología, 16(1), 93-104. https://doi.org/10.1016/S1405-7743(15)72110-4

[2] Nelson, H. (2009). Analysis of the activated sludge model (number 1). EISEVIER. ScienceDirect. Office for official Publications of the European Community, L. (2002). The cost simulation Benchmark: Description and simulator manual. 154.

[3] Laurent, J., Samstag, R. W., Ducoste, J. M., Griborio, A., Nopens, I., Batstone, D. J., ... Potier, O. (2014). A protocol for the use of computational fluid dynamics as a supportive tool for wastewater treatment plant modelling. Water Science and Technology: A Journal of the International Association on Water Pollution Research, 70(10), 1575-1584.

[4] Langeveld, J., Nopens, I., Schilperoort, R., Benedetti, L., Klein, J. de, Amerlinck, Y., \& Weijers, S. (2013). On data requirements for calibration of integrated models for urban water systems. Water Science and Technology, 68(3), 728-736. https://doi.org/10.2166/wst.2013.301

[5] Santa Cruz, J. A., Scenna, N. J., \& Mussati, M. C. (2015). Reducción del modelo ASM1 mediante invariantes de reacción. En IV Argentine Symposium on Industrial Informatics (SII)-JAIIO 44 (Rosario, 2015).

[6]. Akbaş, H., Bilgen, B., \& Turhan, A. M. (2015). An integrated prediction and optimization model of biogas production system at a wastewater treatment facility. Bioresource technology, 196, 566-576.

[7] Sanchez, E. N., Alanís, A. Y., \& Loukianov, A. G. (2008). Discrete-time high order neural control: trained with Kalman filtering (Vol. 112). Springer Science \& Business Media.
[8] V., Alcaraz-González, J., Harmand, A., Rapaport, J. P., Steyer, V., Gonzalez-Alvarez and C., Pelayo-Ortiz, Software sensors for highly uncertain wwtps: a new approach based on interval observers.

Water Research, 36(10), 2515-2524, 2004.

[9] V., Alcaraz-Gonzalez, and V., Gonzalez- Alvarez, Robust nonlinear observers for Bioprocesses: Application to wastewater treatment (Book Chapter in Selected Topics in Dynamics and Control of Chemical and Biological Processes), Springer-Verlag, pp 119-164, Berlin, Germany, 2007.

[10] D., Theilliol, J. C., Ponsart, J., Harmand, C., Join and P., Gras, On-line estimation of unmeasured inputs for anaerobic wastewater treatment processes, Control Engineering Practice, 11(9), 1007-1019, 2003.

[11]. D. A., Urrego-Patarroyo, E. N., Sanchez, S., Carlos-Hernandez and J. F., Beteau, Recurrent neural networks biomass observer for anaerobic processes, IEEE Multi-Conference on Systems and Control, San Antonio, Texas, USA, 2008.

[12]. R., Belmonte-Izquierdo, S., Carlos-Hernández and E. N., Sánchez, A new neural observer for an anaerobic bioreactor, International Journal of Neural Systems, 20(1), 75-86, 2010.

[13] Gurubel, K. J., Osuna-Enciso, V., CoronadoMendoza, A., \& Cuevas, E. (2017). Optimal control strategy based on neural model of nonlinear systems and evolutionary algorithms for renewable energy production as applied to biofuel generation. Journal of Renewable and Sustainable Energy, 9(3), 033101.

[14] Beltramo, T., Ranzan, C., Hinrichs, J., \& Hitzmann, B. (2016). Artificial neural network prediction of the biogas flow rate optimised with an ant colony algorithm. Biosystems Engineering, 143, 68-78.

[15] Akbaş, H., Bilgen, B., \& Turhan, A. M. (2015). An integrated prediction and optimization model of biogas production system at a wastewater 
173

treatment facility. Bioresource technology, 196, 566-576.

[16] A. Y. Alanís, "Entrenamiento de redes neuronales con el filtro de Kalman", Tesis de Maestría, CINVESTAV Unidad Guadalajara, México. 2004.

[17] Sanchez, E. N., Alanís, A. Y., \& Loukianov, A. G. (2008). Discrete-time high order neural control: trained with Kalman filtering (Vol. 112). Springer Science \& Business Media.

[18] Fernández, N., Suárez, C. A., Pérez, E .A., Modelación y Simulación Dinámica para la Gestión de Caudales en la Cuenca Alta del Río Pamplonita -Un Balance Hídrico de Consumo-. Bistua: Revista de la Facultad de Ciencias Básicas [en linea] 2007, 5 (juliodiciembre) : [Fecha de consulta: 8 de mayo de 2018]

Disponible en:<http://www.redalyc.org/articulo.oa?id= 90350208> ISSN 0120-4211

[19] Nelson Fernández, Alberto Ramírez, Fredy Solano.2013. Dinámica Fisicoquímica y Calidad del Agua en la Microcuenca el Volcan, Municipio de Pamplona, Colombia.
Bistua:Revista de la facultad de Ciencias Basicas.2013.3(1):5-17

*Para citar este artículo: Roxana B. Recio Colmenares.; Kelly J. Gurubel Tún.Neuronal modeling of a process of aerobic digestion of wastewater. Revista Bistua.2018.16(1):163-173.

+ Autor para el envió de correspondencia y la solicitud de las separatas: Roxana B. Recio Colmenares Departamento de Estudios del Agua y la Energía, CUTONALA, Universidad de Guadalajara. Ejido San José Tatepozco, Tonalá, Jalisco, 45425, México.joel.gurubel@cutonala.udg.mx, recio.roxana@gmail.com

Recibido: Diciembre 15 de 2016

Aceptado:Febrero 11 de 2018 